University of Nebraska - Lincoln

DigitalCommons@University of Nebraska - Lincoln

Agronomy \& Horticulture -- Faculty Publications

Agronomy and Horticulture Department

2011

Stacking Resistance Alleles from Wild and Domestic Soybean

Sources Improves Soybean Cyst Nematode Resistance

Myungsik Kim

University of Illinois

D. L. Hyten

USDA-ARS, Soybean Genomics and Improvement Laboratory, Beltsville, Maryland, david.hyten@unl.edu

Terry L. Niblack

University of Illinois

Brian W. Diers

University of Illinois, bdiers@illinois.edu

Follow this and additional works at: https://digitalcommons.unl.edu/agronomyfacpub

Part of the Agricultural Science Commons, Agriculture Commons, Agronomy and Crop Sciences Commons, Botany Commons, Horticulture Commons, Other Plant Sciences Commons, and the Plant Biology Commons

Kim, Myungsik; Hyten, D. L.; Niblack, Terry L.; and Diers, Brian W., "Stacking Resistance Alleles from Wild and Domestic Soybean Sources Improves Soybean Cyst Nematode Resistance" (2011). Agronomy \& Horticulture -- Faculty Publications. 795.

https://digitalcommons.unl.edu/agronomyfacpub/795

This Article is brought to you for free and open access by the Agronomy and Horticulture Department at DigitalCommons@University of Nebraska - Lincoln. It has been accepted for inclusion in Agronomy \& Horticulture -Faculty Publications by an authorized administrator of DigitalCommons@University of Nebraska - Lincoln. 


\title{
Stacking Resistance Alleles from Wild and Domestic Soybean Sources Improves Soybean Cyst Nematode Resistance
}

\author{
Myungsik Kim, David L. Hyten, Terry L. Niblack, and Brian W. Diers`
}

\begin{abstract}
The soybean cyst nematode (SCN; Heterodera glycines Ichinohe) is the most economically important soybean [Glycine max (L.) Merr.] pathogen in the United States. Field SCN populations are adapting to the narrowly based SCN resistance currently deployed in soybean cultivars. The objective of our research was to measure the effects of combinations of SCN resistance genes or quantitative trait loci (QTL) from the wild soybean (Glycine soja Siebold \& Zucc.) PI 468916 and the domesticated soybean accessions PI 88788 and PI 437654. Two populations were developed to test the combinations of QTL and genes. Both populations segregated for the G. soja resistance QTL cqSCN-006 and cqSCN-007. Population 1 also segregated for resistance from PI 88788 and Population 2 segregated for resistance from $\mathrm{PI} 437654$. The populations were tested for resistance to three SCN isolates in a greenhouse and with single nucleotide polymorphism (SNP) and simple sequence repeat (SSR) markers. In both populations, the two G. soja resistance alleles significantly increased SCN resistance compared with the alternative alleles. The SCN resistance alleles rhg1 and Rhg4 from PI 437654 and rhg1-b from PI 88788 also significantly increased resistance compared with the alternative alleles. The two G. soja QTL alleles significantly enhanced the resistance derived from $\mathrm{PI} 88788$. These results show that SCN resistance can be increased through stacking genes and QTL from multiple resistance sources.
\end{abstract}

M. Kim, T.L. Niblack, and B.W. Diers, Dep. of Crop Sciences, Univ. of Illinois, Urbana, IL 61801; D.L. Hyten, USDA-ARS, Soybean Genomics and Improvement Lab., Beltsville, MD 20705. Received 6 Aug. 2010. *Corresponding author (bdiers@illinois.edu).

Abbreviations: CTAB, hexadecyltrimethylammonium bromide; EDTA, ethylenediaminetetraacetic acid; FI, female index; $\mathrm{H}^{2}$, broadsense heritability; HG, Heterodera glycines Ichinohe; LG, linkage group; LOD, logarithm of the odds; MQM, multiple QTL model; PCR, polymerase chain reaction; PVC, polyvinyl chloride; QTL, quantitative trait loci; SCN, soybean cyst nematode; SNP, single nucleotide polymorphism; SSR, simple sequence repeat; USLP, Universal Soy Linkage Panel.

Coybean cyst nematode ( $\mathrm{SCN}$ ) is the most damaging pest of soybean in the United States based on yield loss estimates (Wrather and Koenning, 2006). Because of the importance of $\mathrm{SCN}$, there has been focused research on host resistance to this pest. This research has led to the identification of at least 118 SCN resistant soybean plant introductions from the USDA-ARS soybean germplasm collection (Arelli et al., 2000). Some of these resistance sources have been used in breeding programs and resistant cultivars have been available in the United States since 1965 (Brim and Ross, 1966). Although many sources of resistance are available, more than $94 \%$ of the SCN resistant cultivars in Illinois trace their source of resistance to only PI 88788 (Shier, 2008). This wide use of cultivars with resistance from a single source is leading to an increased frequency of SCN populations that can overcome this resistance. In a survey of soil samples collected in fields from Illinois in 2005, it was found that $70 \%$ of the SCN positive samples had populations that could overcome PI 88788 resistance (Niblack et al., 2008).

Published in Crop Sci. 51:934-943 (2011).

doi: 10.2135/cropsci2010.08.0459

Published online 16 Mar. 2011.

(C) Crop Science Society of America | 5585 Guilford Rd., Madison, WI 53711 USA

All rights reserved. No part of this periodical may be reproduced or transmitted in any form or by any means, electronic or mechanical, including photocopying, recording, or any information storage and retrieval system, without permission in writing from the publisher. Permission for printing and for reprinting the material contained herein has been obtained by the publisher. 
The genetic basis of resistance to SCN has been studied and many quantitative trait loci (QTL) controlling this resistance have been mapped (Concibido et al., 2004). In the majority of the sources of resistance studied, the resistance gene rhg1 was mapped as a QTL onto chromosome 18 (formerly linkage group [LG] G). This gene was mapped in the SCN resistance sources PI 437654 (Webb et al., 1995), PI 209332 (Concibido et al., 1996), Peking, PI 90763, PI 88788 (Concibido et al., 1997; Glover et al., 2004), PI 89772 (Yue et al., 2001), PI 88287 (Bassuner et al., 2001), and PI 404198A (Guo et al., 2006). In addition, research showed that PI 437654 and PI 88788 have different alleles at rhg1 (Brucker et al., 2005). The allele from PI 88788 was recently designated rhg1- $b$ by the Soybean Genetics Committee (Kim et al., 2010). rhg1 was detected in most studies with QTL mapping methods because SCN resistance typically segregates quantitatively in populations. Although SCN resistance is quantitatively inherited, rhg1 has a gene designation because it often contributes a sufficiently large effect in populations to be mapped as a Mendelian trait. In this paper, we will use the term gene in reference to rhg1 and a second named and mapped gene Rhg4, but the resistance genes or QTL from Glycine soja Siebold \& Zucc., which are distinct loci from rhg1 and Rhg4, will be referred to as QTL. However, in some cases the terms QTL and gene will be used interchangeably for ease in explanation.

Because of the wide use of PI 88788 as a resistance source, it has been the focus of a number of studies. These mapping studies have revealed the presence of only two resistance QTL that can be traced to this source (Concibido et al., 1997; Glover et al., 2004). This includes rhg1-b on chromosome 18, which also has the confirmed QTL (cq) designation cqSCN-001, and a second QTL on chromosome 16 (formerly LG J), which has the designation cqSCN-003 (Glover et al., 2004).

In contrast to the mapping of only two QTL from PI 88788, many resistance QTL have been mapped from PI 437654 (Webb et al., 1995; Wu et al., 2009). This may explain why PI 437654 is resistant to more HG (Heterodera glycines Ichinohe) types than PI 88788 (Anand et al., 1988; Diers et al., 1997; Niblack et al., 2002). In a recent study, Wu et al. (2009) detected QTL for SCN resistance from PI 437654 on chromosomes 1 (LG D1a), 4 (LG C1), 8 (LG A2), 9 (LG K), 11 (LG B1), 12 (LG H), 18 (LG G), and 20 (LG I) and epistatic interactions mapping to all chromosomes except chromosomes 2 (LG D1b), 9 (LG K), and 14 (LG B2). Consistent with previous studies, the most important resistance allele mapped from PI 437654 was rhg1. This allele was significantly associated with resistance to four of the five SCN isolates tested. In addition, the Rhg4 resistance allele on chromosome 8 was identified as the second most important QTL for two of the five isolates.

The genetic basis of SCN resistance in the G. soja accession PI 468916 has been studied and two resistance QTL were mapped onto chromosomes 15 (LG E) and 18 (LG G) (Wang et al., 2001). These QTL were mapped to unique genetic regions that had not been previously associated with SCN resistance and the chromosome 15 QTL was given the confirmed QTL designation cqSCN-006 and the chromosome 18 QTL the designation cqSCN-007 (available at http:// soybase.org [verified 20 Jan. 2011]). The resistance alleles were then backcrossed into a domestic soybean parent and their positions were further localized (Kabelka et al., 2005). Field studies later showed that the G. soja resistance alleles were not significantly associated with yield drag in fields with low to moderate SCN pressure (Kabelka et al., 2006).

Soybean breeders need new resistance sources, or combinations of resistance genes from different resistance sources, that can provide a more broadly based SCN resistance than is currently available from only PI 88788 . The potential benefits of stacking SCN resistance genes from different sources to broaden host resistance to SCN have not been extensively studied. The objective of our study was to define the effects of SCN resistance genes or QTL from PI 468916, PI 88788, and PI 437654 when combined into common genetic backgrounds. This research specifically focused on cqSCN-006 and cqSCN-007 from G. soja, rhg1-b from PI 88788, and $r h g 1$ and Rhg4 from PI 437654.

\section{MATERIALS AND METHODS}

\section{Population Development}

\section{Population 1}

Population 1 was developed to segregate for $r h g 1-b$ from PI 88788 and G. soja resistance QTL cqSCN-006 and cqSCN-007 from PI 468916. This population is the same as the LDX01-2 population in Kabelka et al. (2006). The population was developed by first backcrossing the two G. soja resistance QTL into the background of the Iowa State University experimental line A81-356022. During each cycle of backcrossing, markers linked to both QTL were used to select the G. soja SCN resistance alleles. An $\mathrm{F}_{1}$ plant developed through three backcrosses $\left(\mathrm{BC}_{3} \mathrm{~F}_{1}\right)$ that carried both G. soja alleles was then crossed with the cultivar Dwight (Nickell et al., 1998), which carries the SCN resistance allele rhg1-b from PI 88788. F $_{1}$ plants produced through this cross were tested with markers linked to the G. soja resistance QTL and an $\mathrm{F}_{1}$ plant heterozygous for both genes was selected. The population was then advanced through single-seed descent to the $\mathrm{F}_{3}$ generation and then advanced to the $F_{3: 6}$ generation by growing each of 81 lines in bulk. When available seed became exhausted for some lines, a single $\mathrm{F}_{6}$ plant was grown from each of the 81 lines and $\mathrm{F}_{6: 7}$ lines from this population were developed.

\section{Population 2}

A second population of $100 \mathrm{~F}_{3 \cdot 4}$ lines (Population 2) was developed from a cross of LD00-2817P with the germplasm line LDX01-1-65 (Diers et al., 2005). LD00-2817P is a high-yielding germplasm line with the SCN resistance sources PI 437654, 
Peking, and PI 88788 in its pedigree (Diers et al., 2010). Marker analysis and the SCN resistance profile of LD00-2817P indicate that it carries rhg1 from either Peking or PI 437654 and not rhg1$b$ from PI 88788. LDX01-1-65 was developed by backcrossing four times cqSCN-006 and cqSCN-007 from PI 468916 into the background of A81-356022. Population 2 was advanced to the $\mathrm{F}_{3}$ generation through single-seed descent and each $\mathrm{F}_{3}$ plant was bulk harvested to derive $100 \mathrm{~F}_{3: 4}$ lines.

\section{Soybean Cyst Nematode Bioassays}

The SCN bioassays were performed individually for each soybean population and SCN isolate under greenhouse conditions from 2007 to 2009. The SCN bioassays were conducted as described by Niblack et al. (2009) using the SCN isolates PA1, PA3, and PA5 that were maintained on the susceptible cultivar Macon at the University of Illinois at Urbana-Champaign. These isolates were originally obtained from Dr. Prakash Arelli, USDA-ARS Mid South Area, Jackson, TN.

For Population 1, the bioassays with the isolates PA1 and PA3 were done by infesting $\mathrm{F}_{6}$ plants from $81 \mathrm{~F}_{3}$-derived lines. For the isolate PA5, the bioassays were done with $\mathrm{F}_{7}$ plants from $81 \mathrm{~F}_{6}$-derived lines. For Population 2, the bioassays for the three isolates were completed by infesting $\mathrm{F}_{4}$ plants from $100 \mathrm{~F}_{3}$-derived lines.

Soybean cyst nematode isolates were increased on Macon and the cysts were crushed to release eggs. The eggs were then collected, transferred to water, centrifuged at $300 \mathrm{~g}$ for $4 \mathrm{~min}$ and layered on the surface of a $45 \%$ sucrose solution. The egg solution was centrifuged at $300 \mathrm{~g}$ for $4 \mathrm{~min}$ and rinsed with water and a sample was counted to estimate the egg numbers.

Seed of the lines being tested were rolled in germination paper and germinated for $3 \mathrm{~d}$ in a growth chamber at $27^{\circ} \mathrm{C}$. The seedlings were transplanted into polyvinyl chloride (PVC) tubes filled with steam-sterilized sandy soil packed into plastic crocks that were suspended in a thermo-regulated water bath. Before transplanting, each PVC tube was infested with approximately 1200 eggs in $1 \mathrm{~mL}$ distilled water with a micropipette. Each PVC tube contained one plant and was an experimental unit. The lines, SCN differentials, and parents were randomized and replicated three times for each isolate. The root systems of plants were maintained at $27 \pm 1^{\circ} \mathrm{C}$ and the plants were grown under a $16-\mathrm{h}$ daylength for $30 \mathrm{~d}$. The plants were then removed from the tubes by gentle soaking in water to loosen soil but not dislodge SCN females. The roots were placed on nested 850$\mu \mathrm{m}$ aperture over $250-\mu \mathrm{m}$ aperture sieves and females were removed from the roots with a water spray and washed into counting dishes. The cysts were counted under the stereomicroscope. Macon was used as the susceptible control to calculate a female index (FI) for each plant with the formula (Golden et al., 1970) FI (\%) = (number of female cyst nematodes on a given individual/average number of female nematodes on Macon) $\times$ 100. Lines were classified as resistant (FI $=0-9)$, moderately resistant $(\mathrm{FI}=10-30)$, moderately susceptible $(\mathrm{FI}=31-60)$, and susceptible (FI > 60) according to Schmitt and Shannon (1992).

\section{DNA Extraction}

Genomic DNA was extracted from a bulk sample of fresh leaf tissue from 10 seedlings of each $\mathrm{F}_{3}$-derived line in both Populations 1 and 2. DNA was also isolated from the $\mathrm{F}_{6}$ plants used to develop the $\mathrm{F}_{6: 7}$ lines in Population 1. DNA was extracted using the hexadecyltrimethylammonium bromide (CTAB) method as described by Saghai Maroof et al. (1984) with slight modifications. Soybean leaf tissue was freeze dried for $48 \mathrm{~h}$ and powdered with a paint shaker for $2 \mathrm{~min}$, and $5 \mathrm{~mL}$ of $1 \mathrm{x}$ CTAB DNA extraction buffer $(50 \mathrm{mM}$ Tris, $\mathrm{pH} 8.0,0.7 \mathrm{M} \mathrm{NaCl}, 10 \mathrm{mM}$ ethylenediaminetetraacetic acid [EDTA], 1\% [w/v] CTAB, and $0.1 \%$ [v/v] 2-mercaptoethanol) was added to the powdered tissue. The solution was incubated for $60 \mathrm{~min}$ at $60^{\circ} \mathrm{C}$ and, after incubation, $5 \mathrm{~mL}$ of chloroform-octanol (24:1) was added and mixed by inversion and centrifuged at $3400 \mathrm{rpm}$ for $10 \mathrm{~min}$. The aqueous phase was removed and the DNA was precipitated by adding 2/3 volume of isopropanol. Precipitated DNA was spooled on a micropipette, transferred to a new tube, washed with $5 \mathrm{~mL}$ of a 70\% ethanol, and then dried at room temperature overnight. Dried DNA was dissolved in 0.1x Tris-EDTA (TE) buffer and used as templates in polymerase chain reactions (PCR).

\section{Genetic Marker Analysis}

The $\mathrm{F}_{3}$ - and $\mathrm{F}_{6}$-derived lines in Population 1 and the $\mathrm{F}_{3}-$ derived lines in Population 2 were evaluated according to Cregan and Quigley (1997) with simple sequence repeat (SSR) markers linked to SCN resistance genes or QTL known to be segregating in the populations. Primer sequences were obtained from the Soybase website (http://soybase.org [verified 10 Jan. 2011]) and the PCR products were sized via electrophoresis on $6 \%$ nondenaturing polyacryamide gels at $340 \mathrm{~V}$ for $2 \mathrm{~h}$ and 20 min (Wang et al., 2003). The products were stained with a 1 ug $\mathrm{mL}^{-1}$ ethidium bromide staining solution for $25 \mathrm{~min}$ and visualized under ultraviolet (UV) light.

The $\mathrm{F}_{6}$-derived lines from Population 1 and the $\mathrm{F}_{3}$-derived lines from Population 2 were evaluated with the Illumina GoldenGate 1536 Universal Soy Linkage Panel 1.0 (USLP 1.0) (Hyten et al., 2010). The GoldenGate assay was performed as per the manufacture's protocol and as described by Hyten et al. (2008) with the sentrix array matrix read on the Illumina BeadStation 500G (Illumina Inc., San Diego, CA). Scoring of the GoldenGate data was accomplished with the Illumina software BeadStudio v.3.2 and visually inspected to ensure that homozygous and heterozygous clusters were properly assigned.

\section{Statistical Analysis}

Broad-sense heritability $\left(\mathrm{H}^{2}\right)$ was calculated for resistance to each isolate in both populations using variance components as described by Fehr (1987). Confidence intervals for the heritability estimates were calculated according to Knapp et al. (1985). The mapping of QTL controlling SCN resistance was completed separately for each population and SCN isolate with the program MapQTL 4.0 (Van Ooijen, 2000; Van Ooijen and Maliepaard, 1996) using the map position of these markers from Hyten et al. (2010). The data were then analyzed with the restricted multiple QTL model (MQM) of MapQTL 4.0, which uses markers as cofactors in a multiple-QTL model. Cofactor markers were selected by the automatic cofactor selection option $(\alpha=0.010)$ to identify a set of cofactors. A marker was used as a cofactor if it was associated with a logarithm of the odds (LOD) score of at least 1.5. If the multifactor analysis decreased the LOD score to below 1.5, that marker was dropped as a cofactor and the analysis was repeated until there was no further change in cofactors. For those QTL known to be 
Table 1. The reaction of three soybean cyst nematode (SCN) isolates to the Heterodera glycines Ichinohe (HG) type differentials and the susceptible cultivar Macon in tests of Population 1 and Population 2.

\begin{tabular}{|c|c|c|c|c|c|c|c|c|c|c|}
\hline \multirow{3}{*}{$\begin{array}{c}\text { Population } \\
\text { test }\end{array}$} & \multirow[b]{3}{*}{ Isolate } & \multirow{3}{*}{$\begin{array}{l}\text { Avg. no. of females } \\
\text { on Macon }\end{array}$} & \multicolumn{7}{|c|}{ Female index ${ }^{\dagger}$} & \multirow[b]{3}{*}{ HG type } \\
\hline & & & 1 & 2 & 3 & 4 & 5 & 6 & 7 & \\
\hline & & & Peking & PI 88788 & PI 90763 & PI 437654 & PI 209332 & PI 89772 & PI 548316 & \\
\hline 1 & PA1 & 78 & 1 & 7 & 0 & 0 & 14 & 0 & 27 & 5.7 \\
\hline 1 & PA3 & 63 & 1 & 2 & 1 & 3 & 9 & 0 & 6 & 0 \\
\hline 1 & PA5 & 408 & 4 & 83 & 0 & 0 & 94 & $\mathrm{ND}^{\ddagger}$ & 87 & 2.5 .7 \\
\hline 2 & PA1 & 101 & 0 & 44 & 0 & 0 & 20 & 0 & 31 & 2.5 .7 \\
\hline 2 & PA3 & 136 & 0 & 28 & 0 & 0 & 35 & 0 & 28 & 2.5 .7 \\
\hline 2 & PA5 & 42 & 0 & 65 & 0 & 0 & 67 & 0 & 81 & 2.5 .7 \\
\hline
\end{tabular}

${ }^{\dagger}$ Female Index $=\left(N_{1} / N_{2}\right) \times 100$, where $N_{1}$ is the average number of females per entry and $N_{2}$ is the average number of females on Macon.

$\ddagger N D$, not determined.

segregating in a population, including rhg1, rhg1-b, Rhg4, cqSCN006, and cqSCN-007, a significance LOD threshold of 2.0 was used. For any other QTL detected in this study, the significance threshold was set to 2.8 for Population 1 and 2.9 for Population 2. These higher thresholds correspond to a genome-wide probability of 0.05 as determined by permutation tests in MapQTL 4.0.

The QTL mapping with MapQTL 4.0 was done for all three SCN isolates in Population 1 with the SSR and single nucleotide polymorphism (SNP) marker data set from the $\mathrm{F}_{6}$-derived lines. These data were used in the mapping although the SCN phenotypes were obtained from $\mathrm{F}_{3}$-derived lines for the SCN isolates PA1 and PA3 while the $\mathrm{F}_{6}$-derived lines were tested with PA5. Analyzing phenotypes collected on $\mathrm{F}_{3}$-derived lines with markers collected on $\mathrm{F}_{6}$-derived lines would have reduced our ability to detect QTL because it is predicted that only one-eighth of the $\mathrm{F}_{3}$-derived lines that were expected to be segregating for any given marker would likely still be segregating in the $\mathrm{F}_{6}$-derived lines. Although this is a concern, we used the $\mathrm{F}_{6}$ marker data in this analysis because SNP marker data were only available for this generation.

The SNP or SSR marker closest to the peak for each significant QTL from the MQM analysis was tested in a multivariate ANOVA with PROC GLM in SAS (SAS Institute, 2001). All two- and three-way interactions between significant markers were tested and those markers that were not significant at $p=0.05$ were removed from the model and the analysis was repeated. An exception to this analysis was the data from PA1 and PA3 on Population 1. For these isolates, single-marker analysis was done in SAS using SSR markers collected from the $\mathrm{F}_{3}$-derived lines and the interactions were tested using SSR markers closely linked to the significant QTL. The $R^{2}$ values from the multivariate models are estimates of the total phenotypic variance explained by the genes or QTL and their interactions. These $R^{2}$ values were used to estimate the proportion of the genotypic variance explained by the QTL using the ratio $R^{2} / \mathrm{H}^{2}$ (Schön et al., 1994).

\section{RESULTS}

\section{Soybean Cyst Nematode Bioassays}

The FI values in the population evaluations and HG type tests were calculated using Macon as a susceptible control instead of the recommended 'Lee 74' (Niblack et al., 2002, 2009). Macon was used because Lee 74 did not germinate in some experiments. Soybean cyst nematode reproduction on Macon varied from a low of 42 cysts lant $^{-1}$ in the test with PA5 in Population 2 to a high of 408 cysts for PA5 in Population 1 (Table 1). The biotypes of the SCN isolates did not respond as expected in the HG type tests. The isolate PA1 was originally defined as a Race 1 isolate (HG type 2-), PA3 was a Race 3 (HG type 0-), and PA5 was a Race 5 (HG type 2-). In our tests, PA1 was an HG type 5.7 (equivalent to a Race 3 or 6 ) or 2.5.7 (Race 1 or 5), PA3 was an HG type 0 (Race 3 or 6 ) or 2.5 .7 (Race 1 or 5 ), and PA5 was an HG type 2.5.7 (Race 1 or 5) (Table 1). It is not unusual to observe changes in SCN HG types as these populations are heterogeneous mixtures of nematodes that can shift to different phenotypes over time (Colgrove et al., 2002).

\section{Results from Soybean Cyst Nematode Tests of Populations Population 1}

The FI of lines in both populations segregated quantitatively with a continuous distribution of FI values (Fig. 1). Population 1 was developed by crossing Dwight, which carries rhg1- $b$ from PI 88788, to a $\mathrm{BC}_{3} \mathrm{~F}_{1}$ plant carrying cqSCN-006 and cqSCN-007. LDX01-1-65, which carries cqSCN-006 and cqSCN-007, was included in the PA1 and PA3 tests of Population 1 and it was found to be moderately susceptible to both isolates (Fig. 1; Table 2). A81-356022, the susceptible recurrent parent used in developing LDX011-65 and the $\mathrm{BC}_{3} \mathrm{~F}_{1}$ parent of Population 1, was susceptible to both isolates with FI values $>100$. Dwight was resistant to PA3 but susceptible to PA1 (FI = 62 for PA1). The FI of parents was not determined for PA5 because these genotypes germinated poorly in this test. Dwight would likely have been susceptible to PA5 because PI 88788, its resistance source, was susceptible to the isolate $(\mathrm{FI}=83)$ (Table 1). The range of FI for lines in Population 1 was 15 to 121 for PA1, 3 to 120 for PA3, and 13 to 114 for PA5 (Fig. 1; Table 2). The $\mathrm{H}^{2}$ of FI ranged from 0.45 to 0.91 (Table 2).

\section{Population 2}

Population 2 was developed by crossing LDX01-1-65 with LD00-2817P. The line LD00-2817P was shown to likely contain rhg1 from PI 437654 or Peking (Diers et al., 2010) and probably Rhg4. In tests with all three isolates, LD00-2817P 
A

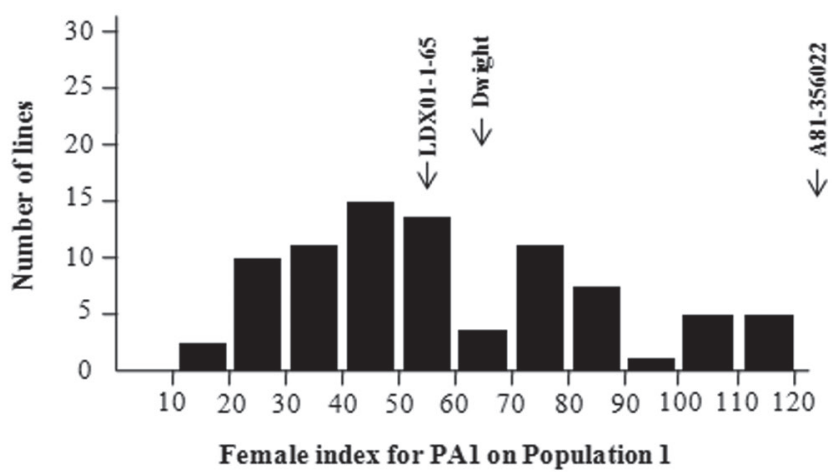

B

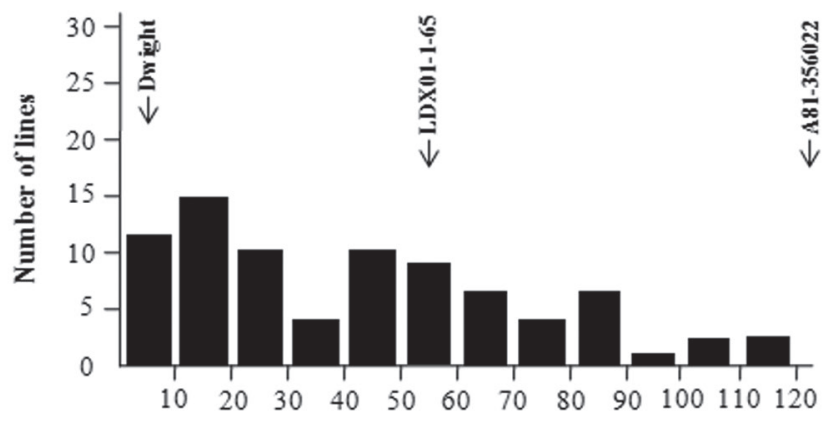

Female index for PA3 on Population 1

C

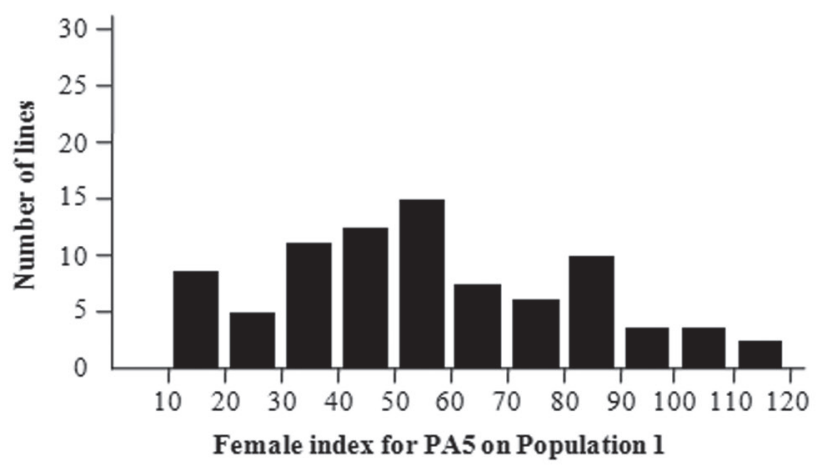

D

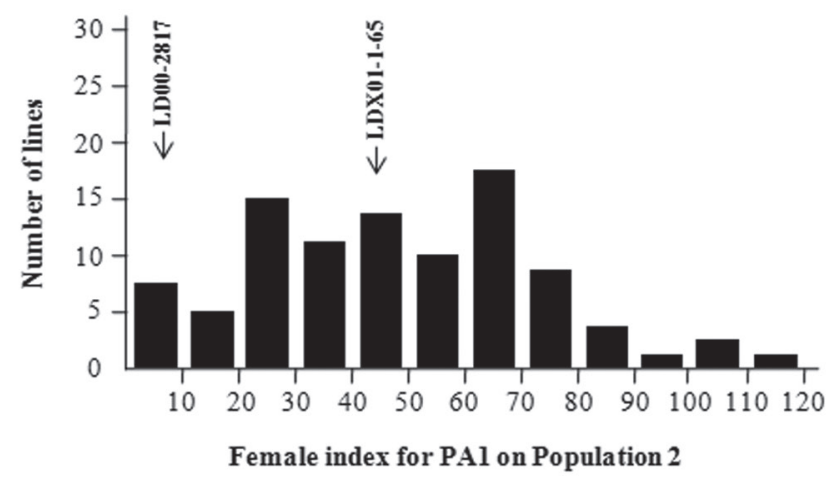

E

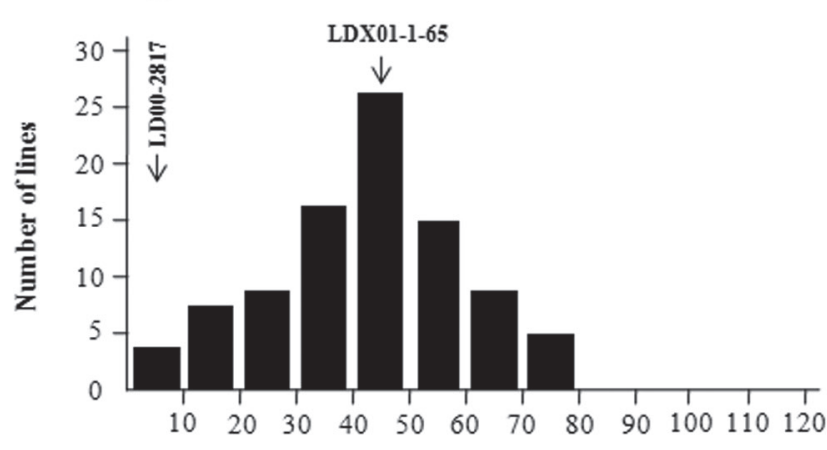

Female index for PA3 on Population 2

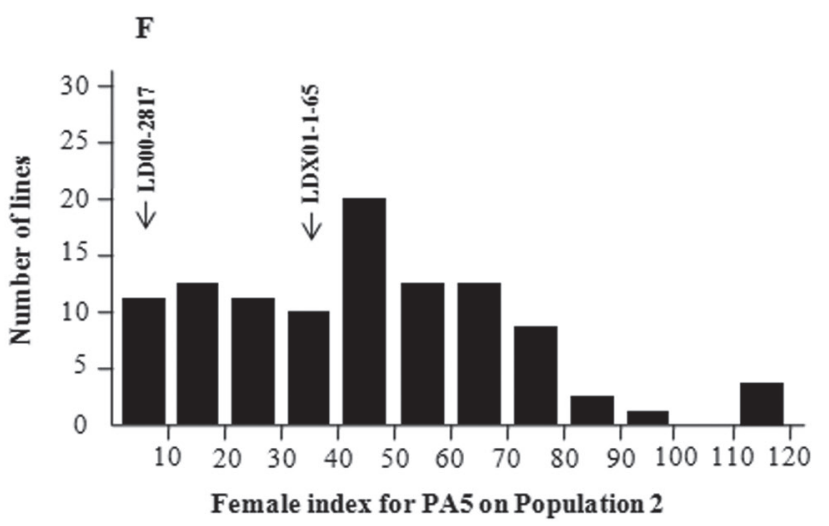

Figure 1. Distribution of female index (FI) for soybean cyst nematode (SCN) isolates PA1, PA3, and PA5 in Population 1, which segregates for resistance from Glycine soja PI 468916 and PI 88788 (A-C), and Population 2, which segregates for resistance from G. soja PI 468916, PI 437654, and Peking (D-F). Parental female index values are indicated by arrow in each distribution except for the PA5 test of Population 1. In this test, the parents germinated poorly.

was rated as resistant and LDX01-1-65 was rated as moderately susceptible (Fig. 1; Table 2). The means of lines in the populations ranged from 0 to 125 for PA1, 4 to 77 for PA3, and 0 to 119 for PA5. The $\mathrm{H}^{2}$ for FI ranged from 0.64 to 0.77 (Table 2).

\section{Detection of Soybean Cyst Nematode Resistance Quantitative Trait Loci}

\section{Population 1}

Quantitative trait loci in Population 1 were mapped with 252 polymorphic SNP markers from the USLP 1.0 and three SSR markers. The population was expected to segregate for the resistance allele $r$ hg1- $b$ from Dwight and resistance alleles at cqSCN-006 and cqSCN-007 originating from G. soja PI 468916. These QTL were detected in different combinations with the MQM analysis for the SCN isolates and no other QTL were detected in the population (Table 3). When QTL were significant, alleles tracing to $G$. soja were associated with greater resistance than the alleles from Dwight for cqSCN-006 and cqSCN007 and the Dwight allele for $r$ h $1-b$ was associated with greater resistance than the allele from the recurrent parent A81-356022. This distribution of effects fit expectations based on the source of the resistance alleles. Although the LOD peaks were not in the same position in the tests for 
Table 2. Estimates of means, ranges, broad-sense heritabilities $\left(\mathrm{H}^{2}\right)$, proportion of the phenotypic variance explained in multiple quantitative trait loci (QTL) models $\left(R^{2}\right)$ with and without the interaction effect, and proportion of genetic variance explained by the QTL model and interactions in Population 1, which segregates for resistance from Glycine soja PI 468916 and PI 88788 , and Population 2, which segregates for resistance from G. soja PI 468916, PI 437654, and Peking.

\begin{tabular}{|c|c|c|c|c|c|c|c|c|c|c|}
\hline \multirow{2}{*}{$\begin{array}{l}\mathrm{SCN}^{\dagger} \\
\text { isolate }\end{array}$} & \multirow{2}{*}{$\begin{array}{l}\text { Population } \\
\text { range }\end{array}$} & \multicolumn{5}{|c|}{ Mean } & \multirow[b]{2}{*}{$\mathrm{H}^{2 \ddagger}$} & \multirow{2}{*}{$\begin{array}{l}R^{2} \text { without } \\
\text { interaction in }\end{array}$} & \multirow{2}{*}{$\begin{array}{c}R^{2} \text { with } \\
\text { nteraction }\end{array}$} & \multirow{2}{*}{$R^{2} / \mathrm{H}^{2 \S}$} \\
\hline & & Population & A81-356022 & Dwight & LDX01-1-65 & LD00-2817P & & & & \\
\hline & & & Female & Index & & 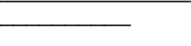 & & & & \\
\hline \multicolumn{11}{|c|}{ Population 1} \\
\hline PA1 & $15-121$ & 57.6 & 152.8 & 61.8 & 53.7 & & $0.45(0.25,0.58)^{q}$ & 0.35 & $N S^{\#}$ & 0.78 \\
\hline PA3 & $3-120$ & 40.7 & 147.2 & 9.6 & 51.5 & & $0.82(0.74,0.87)$ & 0.53 & NS & 0.64 \\
\hline PA5 & $13-114$ & 56.3 & & & & & $0.91(0.87,0.93)$ & 0.74 & 0.75 & 0.82 \\
\hline \multicolumn{11}{|c|}{ Population 2} \\
\hline PA1 & $0-125$ & 47.7 & & & 40.3 & 0 & $0.77(0.68,0.82)$ & 0.63 & 0.73 & 0.95 \\
\hline PA3 & $4-77$ & 43.7 & & & 49.6 & 0.7 & $0.64(0.51,0.75)$ & 0.57 & 0.71 & 1.11 \\
\hline PA5 & $0-119$ & 46.7 & & & 34.7 & 2.4 & $0.73(0.63,0.79)$ & 0.59 & 0.76 & 1.04 \\
\hline
\end{tabular}

†SCN, soybean cyst nematode.

${ }^{\ddagger} \mathrm{H}^{2}$, broad-sense heritability.

\$Proportion of genetic variation explained with the QTL model and interactions.

"Lower and upper 90\% confidence limits in parentheses.

\#NS, no significant interaction.

Table 3. Quantitative trait loci (QTL) for resistance to soybean cyst nematode (SCN) isolates PA1, PA3, and PA5 based on restricted multiple QTL mapping in both Population 1, which segregates for resistance from Glycine soja PI 468916 and PI 88788, and Population 2, which segregates for resistance from G. soja PI 468916, PI 437654, and Peking.

\begin{tabular}{|c|c|c|c|c|c|c|c|}
\hline SCN isolate & Gene or QTL & Chromosome & Marker nearest to the QTL peak & Map position $(\mathrm{cM})^{\dagger}$ & LOD $\ddagger$ & $R^{2}$ & Add. $\S$ \\
\hline \multicolumn{8}{|l|}{ Population 1} \\
\hline \multirow[t]{2}{*}{ PA1 } & cqQTL-006 & 15 & ss107926496 & 78.6 & 3.1 & 12.5 & 9.5 \\
\hline & rhg1-b & 18 & ss107914462 & 11.1 & 3.8 & 15.6 & -10.6 \\
\hline PA3 & rhg1-b & 18 & ss107927739 & 1.6 & 8.6 & 33.5 & -17.3 \\
\hline \multirow[t]{2}{*}{ PA5 } & cqQTL-006 & 15 & ss107926496 & 78.6 & 15.6 & 34.2 & 15.3 \\
\hline & cqQTL-007 & 18 & ss107912619 & 78.3 & 17.3 & 40.0 & 17.4 \\
\hline \multicolumn{8}{|l|}{ Population 2} \\
\hline \multirow[t]{5}{*}{ PA1 } & Rhg4 & 8 & ss107915471 & 46.4 & 5.4 & 10.1 & -17.2 \\
\hline & cqQTL-006 & 15 & Satt491 & 75.6 & 5.0 & 9.1 & 9.4 \\
\hline & rhg1 & 18 & ss107921425 & 9.9 & 9.4 & 19.2 & -15.3 \\
\hline & cqQTL-007 & 18 & ss107912912 & 78.5 & 9.8 & 20.3 & 13.7 \\
\hline & & 16 & ss107920706 & 25.3 & 3.3 & 5.8 & 7.7 \\
\hline \multirow[t]{4}{*}{ PA3 } & Rhg4 & 8 & ss107925847 & 49.3 & 2.1 & 6.6 & -4.2 \\
\hline & cqQTL-006 & 15 & ss107913808 & 77.7 & 3.2 & 10.1 & 5.6 \\
\hline & rhg1 & 18 & ss107914420 & 10.4 & 4.4 & 14.6 & -8.0 \\
\hline & cqQTL-007 & 18 & ss107913107 & 73.3 & 7.5 & 28.2 & 9.3 \\
\hline \multirow[t]{3}{*}{ PA5 } & cqQTL-006 & 15 & ss107913808 & 77.7 & 9.6 & 23.1 & 16.1 \\
\hline & rhg1 & 18 & Satt309 & 10.1 & 3.2 & 6.5 & -9.2 \\
\hline & cqQTL-007 & 18 & ss107912912 & 78.5 & 11.6 & 29.4 & 17.6 \\
\hline
\end{tabular}

†Position of the marker nearest the QTL peak on the single nucleotide polymorphism (SNP) map (Hyten et al., 2010).

‡LOD, logarithm of the odds.

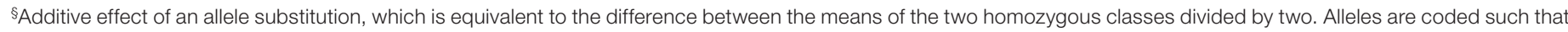

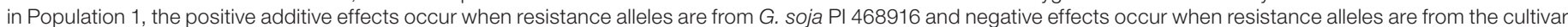

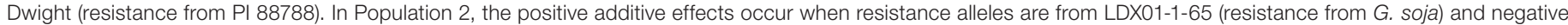
effects occur when resistance alleles are from the cultivar LD00-2817P (resistance from PI 437654, Peking, and PI88788).

each isolate (Table 3), the peaks were sufficiently close to indicate that the same QTL were likely conferring resistance to each SCN isolate for which they were significant.

For PA1, markers linked to cqSCN-006 and rhg1-b were significant in the MQM analysis (Table 3) using the SNP and SSR marker data from the $\mathrm{F}_{6}$-derived lines and the SCN FI from the $\mathrm{F}_{3}$-derived lines. When SSR marker data from the $\mathrm{F}_{3}$-derived lines linked to the three known
SCN resistance genes segregating in the population were used in a single-marker analysis with SAS, cqSCN-007 was also significant $(p<0.006)$. In the multivariate model, no significant interaction $(p=0.05)$ was detected between these three significant loci and together they explained $35 \%$ of the total variance for resistance (Table 2). Lines in the population homozygous for the three susceptibil- 
ity alleles had a FI of 74.5 compared to a mean of 28.1 for lines homozygous for the three resistance alleles.

Only rhg1- $b$ was significant for PA3 in the MQM analysis with the SNP data from the $\mathrm{F}_{6}$-derived lines and the FI values from the $\mathrm{F}_{3}$-derived lines. rhg1- $b$ was also the only QTL or gene significant when the single-marker analysis was done in SAS using the SSR markers from the $\mathrm{F}_{3}$-derived lines that were used to collect the FI values. This QTL was associated with 53\% of the total variance for resistance in this population when the marker data from the $\mathrm{F}_{3}$-derived lines were used (Table 2). Those lines homozygous for the resistance allele had a FI of 15.8 compared to 64.4 for homozygous susceptible lines.

The $\mathrm{F}_{6}$-derived lines were tested for resistance to PA5 and both cqSCN-006 and cqSCN-007 were significant in the MQM analysis for this isolate, but $r h g 1-b$ was not. It was expected that $r$ gr 1- $b$ would not be significant given that PI 88788 was susceptible to the isolate. When the two significant QTL were included in a multivariate model, a significant interaction was detected. The two QTL and their interaction explained $75 \%$ of the total variation for resistance to the isolate (Table 2). Those lines homozygous for the two resistance alleles had a FI of 24.0 compared with a mean FI of 88.7 for the homozygous susceptible lines.

\section{Population 2}

Population 2 segregated for 390 SNP markers from the USLP 1.0 and the data from these SNP markers and three SSR markers were used to map QTL. The population was expected to segregate for cqSCN-006, cqSCN-007, rhg1, and $R h g 4$ based on parentage. In contrast to Population 1, the two G. soja QTL and rhg1 were significantly associated with resistance to each of the three isolates in the MQM analysis (Table 3). Consistent with expectations, the allele conferring increased resistance was from LD00-2817P for rhg1 and Rhg4 and from LDX01-1-65 for cqSCN-006 and cqSCN-007.

For PA1, cqSCN-006, cqSCN-007, rhg1, Rhg4, and a new QTL on chromosome 16 (formerly LG J) were significant in the MQM analysis (Table 3). The chromosome 16 resistance allele was derived from LDX01-1-65 and it maps to a region where no other SCN resistance QTL have been reported in the literature (http://soybase. org [verified 10 Jan. 2011]). To determine the origin of the chromosome 16 resistance allele, the recurrent parent A81-356022 and the donor parent PI 468916 were compared to LDX01-1-65 with the 1536 markers in USLP 1.0. There was no evidence of introgression of regions on chromosome 16 from PI 468916 into LDX01-1-65, indicating that the resistance allele on this chromosome originated from A81-356022 and not PI 468916.

The $R^{2}$ values for the PA1 resistance genes and QTL ranged from 20.3 (cqSCN-007) to 5.8 (chromosome 16 QTL). When markers linked to these genes and QTL and all possible two-way interactions were added to a multivariate model (Table 2), significant interactions between Rhg4 and rhg1 and between Rhg4 and cqSCN006 were detected. The significant markers and the two significant interactions explained $73 \%$ of the total variation for resistance in the multivariate model (Table 2). There were no lines in the population that carried all five alleles conferring resistance or susceptibility; however, those lines that carried four resistance alleles had FI averaging 4.9 and those with four susceptibility alleles averaged 79.0.

Rhg4, rhg1, cqSCN-006, and cqSCN-007 were all significant for PA3 in the MQM analysis (Table 3). The multivariate SAS model that included the genes and QTL and all two-way interactions revealed only one significant interaction: between rhg1 and cqSCN-006. The genes and QTL together with the significant interaction explained $71 \%$ of the total variation for resistance (Table 2). Those lines that carried three or four resistance alleles had a FI averaging 23.3 and those with three or four susceptibility alleles had a FI of 57.6.

For PA5, cqSCN-006 and cqSCN-007 and rhg1 were significant in addition to two-way interactions between cqSCN-006 and cqSCN-007 and between rhg1 and cqSCN007 and a three-way interaction between the three loci. The combination of the two QTL, rhg1, and their interactions explained $76 \%$ of the total variation for resistance. Those lines with three resistance alleles had an FI of 14.3 and those with three susceptibility alleles had an FI of 92.1.

\section{DISCUSSION}

The populations used in these experiments were developed to test the effects of cqSCN-006 and cqSCN-007 in combination with rhg1-b from PI88788 and with $r h g 1$ and Rhg 4 from PI 437654 or Peking. These QTL and genes were all detected in the current study showing the effectiveness of cqSCN-006 and cqSCN-007 in combination with genes from these other resistance sources.

Because the main objective of this study was to test these new combinations of known QTL that have fairly large effects, we used relatively small populations. The employment of these small populations may have resulted in the inability to detect some small-effect QTL segregating in these populations.

The locations of rhg1, rhg1-b, Rhg4, cqSCN-006, and cqSCN-007 were mapped in the current study and matched their positions reported in previous studies. Cregan et al. (1999) and Kim et al. (2010) reported that the SSR marker Satt309 mapped within $1 \mathrm{cM}$ on the proximal (centromeric) side of $r h g 1$ and both $r h g 1$ and $r h g 1-b$ mapped within $8.5 \mathrm{cM}$ of Satt309 in the current study. Rhg4 was previously mapped near the $i$ locus (Matson and Williams, 1965; Webb et al., 1995), which is at cM position 48 on the 2003 composite soybean map (http://soybase.org [verified 10 Jan. 2011]). In Population 2, Rhg4 was mapped within 
$2 \mathrm{cM}$ of this position. Both cqSCN-006 and cqSCN-007 also mapped close to the regions where they were positioned by Kabelka et al. (2005).

A previously undetected QTL was mapped in the current study to chromosome 16 in Population 2. There are previous reports of a SCN resistance QTL on chromosome 16, which includes the confirmed resistance QTL cqSCN-003 originating from PI 88788 (Glover et al., 2004). The region where cqSCN-003 maps was previously identified to have resistance QTL from PI 90763 (Guo et al., 2005; Concibido et al., 1997) and PI 209332 (Concibido et al., 1994). The QTL detected in Population 2 maps more than $40 \mathrm{cM}$ from cqSCN-003 to a region where there are no previously mapped SCN resistance QTL listed on the Soybase website (http://soybase. org [verified 10 Jan. 2011]), suggesting that this is a newly identified SCN resistance QTL.

Both Population 1 and 2 were developed using backcross lines that have A81-356022 as the recurrent parent; however, the chromosome 16 QTL was mapped in Population 2 but not in Population 1. The marker analysis showed that the resistance allele for this QTL originated from A81356022 in Population 2, so this resistance QTL should have been present in both populations. These results suggest that there is background specificity in the effect of the QTL. Alternatively, although the chromosome 16 QTL is significant with a genome-wide threshold of 0.05 , it is also possible that the detection of this QTL was a type 1 error.

In contrast to detecting only the effects of rhg1 and Rhg4 from LD00-2817P, our parent with resistance tracing to PI 437654, Wu et al. (2009) detected SCN resistance genes and QTL at nine locations in the genome from PI 437654 using five SCN isolates. Of these genes and QTL, generally rhg1 and Rhg4 had the largest effects although a QTL on chromosome 11 (LG B1) had a large effect for the Race 5 isolate used in the study. In contrast to $\mathrm{Wu}$ et al. (2009), who developed their population by directly crossing PI 437654 to a susceptible line, we developed our population using the elite germplasm line LD00-2817P as a parent. Minor SCN resistance QTL in PI 437654 were especially likely to be lost during the breeding process resulting in LD00-2817P not carrying the full complement of genes and QTL detected by Wu et al. (2009). Evidence of this loss is that Ina, the parent LD00-2817 inherited its resistance from PI 437654, was reported to not have full resistance to SCN Race 14 (Nickell et al., 1999). In contrast, PI 437654 was shown to have complete resistance to this race (Diers et al., 1997). In addition, Diers et al. (2010) found that LD00-2817P generally had greater FI values than PI 437654 in a test with 12 SCN isolates. Another possible reason for not identifying minor QTL from PI 437654 is that Population 2 was composed of $100 \mathrm{~F}_{3}$-derived lines compared with 205 lines in the population used by Wu et al. (2009). The small size of our population would have reduced our ability to detect minor QTL if they were present in LD00-2817P.

Significant interactions were observed between significant QTL and genes. The interaction observed between Rhg4 and rhg1 was previously reported by Wu et al. (2009), Brucker et al. (2005), and Webb et al. (1995) for these genes from PI 437654 and by Meksem et al. (2001) for these genes from Peking. The interaction we observed between Rhg4 and rhg1 was SCN isolate specific as it was detected for PA1 but not PA3 or PA5. The SCN isolate specificity of this interaction was also observed by Brucker et al. (2005) and Wu et al. (2009). Brucker et al. (2005) observed a significant interaction between rhg1 and Rhg4 for SCN isolate PA3, which was shown to have a HG Type 0 or 7 in that study, but not the isolate TN13, which had a HG Type 1.2.5.7. Wu et al. (2009) detected a significant interaction between Rhg4 and rhg1 for Race 1 (HG type 2.5.7) and Race 3 (HG type 0) isolates but not for Race 2 (HG type 1.2.5.7), 5 (HG type 2.7), and 14 (HG type 1.3.5.6.7) isolates.

The heritabilities for resistance varied from a low of 0.45 for PA1 in Population 1 to a high of 0.91 for PA5 in Population 1. Although the PA5 test for Population 1 did have the greatest number of females on the susceptible cultivar Macon for any of the tests, there was no clear association between reproduction on Macon and the heritability of the test. For example, the PA5 test for Population 2 had the least Macon reproduction but had a heritability of 0.73 .

The proportion of the genetic variance explained by the QTL and interactions ranged from 0.77 to greater than 1, showing that the mapped genes control most of the genetic variance for resistance (Table 2). The values of greater than 1 obtained for PA 3 and PA5 in Population 2 may be the result of our small population sizes, which can lead to an overestimate of $R^{2}$ values (Beavis, 1994). A second explanation is that the heritability values were underestimated, which is possible given the fairly large standard errors of the heritability estimates (Table 2).

In general, there was more value in combining cqSCN006 and cqSCN-007 with PI 88788 resistance than with the PI 437654 or Peking resistance in Population 2. The usefulness of the G. soja QTL alleles in the PI 88788 background is shown by Dwight not being resistant to PA1 and PI 88788 not being resistant to PA5 (Table 1; Fig. 1). The deficiencies in the PI 88788 resistance to these isolates were at least partially offset by the addition of cqSCN-006 and cqSCN-007. We observed in Population 2 a significant effect of the two G. soja QTL; however, LD00-2817P, the parent with resistance tracing to PI 437654 and Peking, was already resistant to the three SCN isolates used in the study (Fig. 1; Table 2). When lines with different gene and QTL combinations in Population 2 were compared after inoculation with PA1, lines with the resistance alleles at rhg1 and Rhg4 and the susceptibility alleles at both G. soja alleles had a FI of 2.6, 
whereas lines with the resistance allele for the four genes and QTL had a FI of 0.0, showing little advantage of the G. soja alleles. In contrast, there was an advantage of combining the G. soja alleles with rhg1 for resistance to PA5 (Rhg4 was not significant for this isolate). Lines that had only the resistance allele at rhg1 had a FI of 55.1, whereas lines with $r$ gr 1 and the two G. soja alleles had a FI of 14.4.

As stated previously, most SCN resistant cultivars in the midwestern United States have their SCN resistance from PI 88788. The widespread use of this resistance source is at least partially the result of the ability of breeders to combine high yield with this source of resistance. A strategy that breeders may want to consider is combining the PI 88788 resistance with cqSCN-006 and cqSCN007. This will provide a unique resistance source with a broad diversity of resistance alleles. Although this combination does not give as high a level of resistance as PI 437654 , it would give broad-based partial resistance that could help protect yields.

\section{Acknowledgments}

This work was supported by soybean check-off funding from the Illinois Soybean Association and the USDA CSREES grant \#ILLU-483-614, 2006-34488-16915 to BD.

\section{References}

Anand, S.C., K.M. Gallo, I.A. Baker, and E.E. Hartwig. 1988. Soybean plant introductions with resistance to Races 4 and 5 of soybean cyst nematodes. Crop Sci. 28:563-564.

Arelli, P.R., D.A. Sleper, P. Yue, and J.A. Wilcox. 2000. Soybean reaction to races 1 and 2 of Heterodera glycines. Crop Sci. 40:824-826.

Bassuner, R., K. Clayton, K.S. Lewers, S. Mackenzie, R.C. Shoemaker, and Z. Vaghchhipawala. 2001. Modulations in gene expression and mapping of genes associated with cyst nematode infection of soybean. Mol. Plant Microbe Interact. 14:42-54.

Beavis, W.D. 1994. The power and deceit of QTL experiments: Lessons from comparative QTL studies. p. 250-266. In Proc. Annu. Corn Sorghum Res. Conf., 49th, Chicago, IL. 7-8 Dec. 1994 Am. Seed Trade Assoc., Washington, DC.

Brim, C., and J.P. Ross. 1966. Registration of Pickett soybeans. Crop Sci. 6:305.

Brucker, E., S. Carlson, E. Wright, T. Niblack, and B. Diers. 2005. Rhg1 alleles from soybean PI 437654 and PI 88788 respond differentially to isolates of Heterodera glycines in the greenhouse. Theor. Appl. Genet. 111:44-49.

Cregan, P.B., J. Mudge, E.W. Fickus, D. Danesh, R. Denny, and N.D. Young. 1999. Two simple sequence repeat markers to select for soybean cyst nematode resistance conditioned by the rhg1 locus. Theor. Appl. Genet. 99:811-818.

Cregan, P.B., and C.V. Quigley. 1997. Simple sequence repeat DNA marker analysis. p. 173-185. In G. Caetano-Anolles et al. (ed.) DNA markers: Protocols, applications and overviews. John Wiley \& Sons, Hoboken, NJ.

Colgrove, A.L., G.S. Smith, J.A. Wrather, R.D. Heinz, and T.L. Niblack. 2002. Lack of predictable race shift in Heterodera glycines-infested field plots. Plant Dis. 86:1101-1108.
Concibido, V.C., R.L. Denny, S.R. Boutin, R. Hautea, J.H. Orf, and N.D. Young. 1994. DNA marker analysis of loci underlying resistance to soybean cyst nematode (Heterodera glycines Ichinohe). Crop Sci. 34:240-246.

Concibido, V.C., R.L. Denny, D.A. Lange, J.H. Orf, and N.D. Young. 1996. RFLP mapping and marker-assisted selection of soybean cyst nematode resistance in PI 209332. Crop Sci. 36:1643-1650.

Concibido, V.C., B.W. Diers, and P.R. Arelli. 2004. A decade of QTL mapping for cyst nematode resistance in soybean. Crop Sci. 44:1121-1131.

Concibido, V.C., D.A. Lange, R.L. Denny, J.H. Orf, and N.D. Young. 1997. Genome mapping of soybean cyst nematode resistance genes in 'Peking', PI 90763 and PI 88788 using DNA markers. Crop Sci. 37:258-264.

Diers, B.W., P.R. Arelli, S.R. Carlson, W.R. Fehr, E.A. Kabelka, R.C. Shoemaker, and D. Wang. 2005. Registration of “LDX01-1-65' soybean. Crop Sci. 45:1671.

Diers, B.W., T. Cary, D. Thomas, A. Colgrove, and T. Niblack. 2010. Registration of LD00-2817P soybean germplasm line with resistance to soybean cyst nematode from PI 437654. J. Plant Reg. 4:141-144.

Diers, B.W., H.T. Skorupska, A.P. Rao-Arelli, and S.R. Cianzio. 1997. Genetic relationships among soybean plant introductions with resistance to soybean cyst nematodes. Crop Sci. 37:1966-1972.

Fehr, W.R. 1987. Principles of cultivar development. Vol. 1. Theory and technique. Macmillan, New York, NY.

Glover, K.D., D. Wang, P.R. Arelli, S.R. Carlson, S.R. Cianzio, and B.W. Diers. 2004. Near isogenic lines confirm a soybean cyst nematode resistance gene from PI 88788 on linkage group J. Crop Sci. 44:936-941.

Golden, A.M., J.M. Epps, R.D. Riggs, L.A. Duclos, J.A. Fox, and R.L. Bernard. 1970. Terminology and identification of intraspecific forms of the soybean cyst nematode (Heterodera glycines). Plant Dis. Rep. 54:544-546.

Guo, B., D.A. Sleper, P.R. Arelli, J.G. Shannon, and H.T. Nguyen. 2005. Identification of QTLs associated with resistance to soybean cyst nematode races 2, 3 and 5 in soybean PI 90763. Theor. Appl. Genet. 111:965-971.

Guo, B., D.A. Sleper, H.T. Nguyen, P.R. Arelli, and J.G. Shannon. 2006. Quantitative trait loci underlying resistance to three soybean cyst nematode populations in soybean PI 404198A. Crop Sci. 46:224-233.

Hyten, D.L., I.-Y. Choi, Q. Song, J.E. Specht, T.E. Carter, R.C. Shoemaker, E.-Y. Hwang, L.K. Matukumalli, and P.B. Cregan. 2010. A high density integrated genetic linkage map of soybean and the development of a 1536 Universal Soy Linkage Panel for QTL mapping. Crop Sci. 50:960-968.

Hyten, D.L., Q. Song, I.Y. Choi, M.S. Yoon, J.E. Specht, L.K. Matukumalli, R.L. Nelson, R.C. Shoemaker, N.D. Young, and P.B. Cregan. 2008. High-throughput genotyping with the GoldenGate assay in the complex genome of soybean. Theor. Appl. Genet. 116:945-952.

Kabelka, E.A., S.R. Carlson, and B.W. Diers. 2005. Marker saturation and the localization of two soybean cyst nematode resistance loci from Glycine soja PI 468916. Crop Sci. 45:2473-2481.

Kabelka, E.A., S.R. Carlson, and B.W. Diers. 2006. Glycine soja PI 468916 SCN Resistance loci's associated effects on soybean seed yield and other agronomic traits. Crop Sci. 46:622-629.

Kim, M., D.L. Hyten, A.F. Bent, and B.W. Diers. 2010. Fine mapping of the SCN resistance locus rhg1-b from PI 88788. Plant Gen. 
3(2):81-89.

Knapp, S.J., W.W. Stroup, and W.M. Ross. 1985. Exact confidence intervals for heritability on a progeny mean basis. Crop Sci. 25:192-194.

Matson, A.L., and L.F. Williams. 1965. Evidence of a fourth gene for resistance to the soybean cyst nematode. Crop Sci. 5:477.

Meksem, K., P. Pantazopoulos, V.N. Njiti, L.D. Hyten, P.R. Arelli, and D.A. Lightfoot. 2001. 'Forrest' resistance to the soybean cyst nematode is bigenic: Saturation mapping of the rhg1 and Rhg4 loci. Theor. Appl. Genet. 103:710-717.

Niblack, T.L., P.R. Arelli, G.R. Noel, C.H. Opperman, J.H. Orf, D.P. Schmitt, J.G. Shannon, and G.L. Tylka. 2002. A revised classification scheme for genetically diverse populations of Heterodera glycines. J. Nematol. 34:279-288.

Niblack, T.L., A.L. Colgrove, K. Colgrove, and J.P. Bond. 2008. Shift in virulence of soybean cyst nematode is associated with use of resistance from PI 88788. Plant Health Prog. doi:10.1094/PHP-2008-0118-01-RS.

Niblack, T., G.L. Tylka, P. Arelli, J. Bond, B. Diers, P. Donald, J. Faghihi, V.R. Ferris, K. Gallo, R.D. Heinz, H. LopezNicora, R. Von Qualen, T. Welacky, and J. Wilcox. 2009. A standard greenhouse method for assessing soybean cyst nematode resistance in soybean: SCE08 (Standardized Cyst Evaluation 2008). Plant Health Prog. 10.1094/PHP-20090513-01-RV.

Nickell, C.D., G.R. Noel, T.R. Cary, and D.J. Thomas. 1998. Registration of 'Dwight' soybean. Crop Sci. 38:1398.

Nickell, C.D., G.R. Noel, T.R. Cary, D.J. Thomas, and R.A. Leitz. 1999. Registration of 'Ina' soybean. Crop Sci. 39:1533.

Saghai-Maroof, M.A., K.M. Soliman, R.A. Jorgensen, and R.W. Allard. 1984. Ribosomal DNA spacer-length polymorphisms in barley: Mendelian inheritance, chromosomal location and population dynamics. Proc. Natl. Acad. Sci. USA 81:8014-8018.

SAS Institute. 2001. Statistical analysis software (SAS): Users guide. SAS Inst., Cary, NC.

Schmitt, D.P., and G. Shannon. 1992. Differentiating soybean responses to Heterodera glycines races. Crop Sci. 32:275-277.

Schön, C.C., A.E. Melchinger, J. Boppenmaier, E. BrunklausJung, R.G. Herrmann, and J.F. Seitzer. 1994. RFLP mapping in maize: Quantitative trait loci affecting testcross performance of elite European flint lines. Crop Sci. 34:378-389.

Shier, M. 2008. Soybean varieties with soybean cyst nematode resistance. Available at https://netfiles.uiuc.edu/tjw/www/ cover.htm (verified 10 Jan. 2011). Livingston County Extension Unit, Univ. of Illinois Extension, Pontiac, IL.

Van Ooijen, J.W. 2000. MapQTL version 4.0: User friendly power in QTL mapping. Addendum to the manual of version 3.0. Plant Research International, Wageningen, The Netherlands.

Van Ooijen, J.W., and C. Maliepaard. 1996. MapQTL version 3.0: Software for the calculation of QTL positions on genetic maps. CPRO-DLO, Wageningen, The Netherlands.

Wang, D., P.R. Arelli, R.C. Shoemaker, and B.W. Diers. 2001. Loci underlying resistance to Race 3 of soybean cyst nematode in Glycine soja plant introduction 468916. Theor. Appl. Genet. 103:561-566.

Wang, D., J. Shi, S.R. Carlson, P.B. Cregan, R.W. Ward, and B.W. Diers. 2003. A low-cost, high-throughput polyacrylamide gel electrophoresis system for genotyping with microsatellite DNA markers. Crop Sci. 43:1828-1832.

Webb, D.M., B.M. Baltazar, A.P. Rao-Arelli, J. Schupp, K. Clayton, P. Keim, and W.D. Beavis. 1995. Genetic mapping of soybean cyst nematode race-3 resistance loci in soybean PI 437.654. Theor. Appl. Genet. 91:574-581.

Wrather, J.A., and S.R. Koenning. 2006. Estimates of disease effects on soybean yields in the United States 2003 to 2005. J. Nematol. 38:173-180.

Wu, X., S. Blake, D.A. Sleper, J.G. Shannon, P. Cregan, and H.T. Nguyen. 2009. QTL, additive and epistatic effects for SCN resistance in PI 437654. Theor. Appl. Genet. 118:1093-1105.

Yue, P., D.A. Sleper, and P.R. Arelli. 2001. Mapping resistance to multiple races of Heterodera glycines in soybean PI 89772. Crop Sci. 41:1589-1595. 


\section{Stacking Resistance Alleles from Wild and Domestic Soybean Sources Improves Soybean Cyst Nematode Resistance}

Myungsik Kim, David L. Hyten, Terry L. Niblack, and Brian W. Diers

In Volume 51, Number 3, pp. 934-943, seven values in the eighth column of Table 3 were incorrect. The corrected table appears below.

Table 3. Quantitative trait loci (QTL) for resistance to soybean cyst nematode (SCN) isolates PA1, PA3, and PA5 based on restricted multiple QTL mapping in both Population 1, which segregates for resistance from Glycine soja PI 468916 and PI 88788, and Population 2, which segregates for resistance from G. soja PI 468916, PI 437654, and Peking.

\begin{tabular}{|c|c|c|c|c|c|c|c|}
\hline SCN isolate & Gene or QTL & Chromosome & Marker nearest to the QTL peak & Map position $(\mathrm{cM})^{\dagger}$ & LOD $^{\ddagger}$ & $R^{2}$ & Add. ${ }^{\S}$ \\
\hline \multicolumn{8}{|l|}{ Population 1} \\
\hline \multirow[t]{2}{*}{ PA1 } & cqQTL-006 & 15 & ss107926496 & 78.6 & 3.1 & 12.5 & 9.5 \\
\hline & rhg1-b & 18 & ss107914462 & 11.1 & 3.8 & 15.6 & -10.6 \\
\hline PA3 & rhg1-b & 18 & ss107927739 & 1.6 & 8.6 & 33.5 & -17.3 \\
\hline \multirow[t]{2}{*}{ PA5 } & cqQTL-006 & 15 & ss107926496 & 78.6 & 15.6 & 34.2 & 15.3 \\
\hline & cqQTL-007 & 18 & ss107912619 & 78.3 & 17.3 & 40.0 & 17.4 \\
\hline \multicolumn{8}{|l|}{ Population 2} \\
\hline \multirow[t]{5}{*}{ PA1 } & Rhg4 & 8 & ss107915471 & 46.4 & 5.4 & 10.1 & -17.2 \\
\hline & cqQTL-006 & 15 & Satt491 & 75.6 & 5.0 & 9.1 & 9.4 \\
\hline & rhg1 & 18 & ss107921425 & 9.9 & 9.4 & 19.2 & -15.3 \\
\hline & cqQTL-007 & 18 & ss107912912 & 78.5 & 9.8 & 20.3 & 13.7 \\
\hline & & 16 & ss107920706 & 25.3 & 3.3 & 5.8 & 7.7 \\
\hline \multirow[t]{4}{*}{ PA3 } & Rhg4 & 8 & ss107925847 & 49.3 & 2.1 & 6.6 & -4.2 \\
\hline & cqQTL-006 & 15 & ss107913808 & 77.7 & 3.2 & 10.1 & 5.6 \\
\hline & rhg1 & 18 & ss107914420 & 10.4 & 4.4 & 14.6 & -8.0 \\
\hline & cqQTL-007 & 18 & ss107913107 & 73.3 & 7.5 & 28.2 & 9.3 \\
\hline \multirow[t]{3}{*}{ PA5 } & cqQTL-006 & 15 & ss107913808 & 77.7 & 9.6 & 23.1 & 16.1 \\
\hline & rhg1 & 18 & Satt309 & 10.1 & 3.2 & 6.5 & -9.2 \\
\hline & cqQTL-007 & 18 & ss107912912 & 78.5 & 11.6 & 29.4 & 17.6 \\
\hline
\end{tabular}

†Position of the marker nearest the QTL peak on the single nucleotide polymorphism (SNP) map (Hyten et al., 2010).

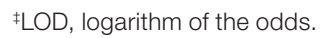

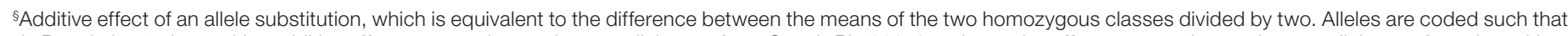

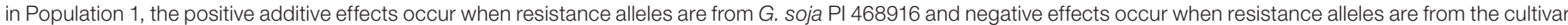

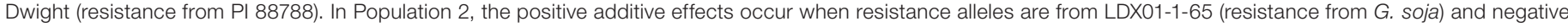
effects occur when resistance alleles are from the cultivar LD00-2817P (resistance from PI 437654, Peking, and PI88788).

Published in Crop Sci 51:2301 (2011).

doi: $10.2135 /$ cropsci2010.08.0459er

(C) Crop Science Society of America | 5585 Guilford Rd., Madison, WI 53711

All rights reserved. No part of this periodical may be reproduced or transmitted in any form or by any means, electronic or mechanical, including photocopying, recording, or any information storage and retrieval system, without permission in writing from the publisher. Permission for printing and for reprinting the material contained herein has been obtained by the publisher. 